\title{
INCREASING EXPRESSION OF HEPATITIS B SURFACE ANTIGEN IN MAIZE THROUGH BREEDING
}

\author{
A Thesis \\ Presented to \\ the Faculty of California Polytechnic State University, \\ San Luis Obispo
}

\begin{abstract}
In Partial Fulfillment
of the Requirements for the Degree

Master of Agriculture with a specialization in Crop Science
\end{abstract}

By

Erin Suzanne Miller

March 2015 
(C) 2015

Erin Suzanne Miller

ALL RIGHTS RESERVED 
COMMITTEE MEMBERSHIP

TITLE:

Increasing Expression of Hepatitis B Surface Antigen in

Maize through Breeding

AUTHOR:

Erin Suzanne Miller

DATE SUBMITTED:

March 2015

COMMITTEE CHAIR:

Jeffrey C. Wong, Ph.D.

Professor Horticulture and Crop Science Department

COMMITTEE MEMBER: John Howard, Ph.D.

President of the Applied Biotechnology Institute

COMMITTEE MEMBER: Celine Hayden, Ph.D.

Director of Research Development at the Applied

Biotechnology Institute 


\begin{abstract}
Increasing Expression of Hepatitis B Surface Antigen in Maize through Breeding Erin Suzanne Miller
\end{abstract}

The hepatitis B virus (HBV) is a common virus, with two billion people infected worldwide. It causes approximately 600,000 deaths each year, despite the availability of an effective vaccine since 1982. Maize as a platform for oral vaccination can supply a heat stable vaccine, which does not require syringes or trained personnel to administer. The Hepatitis B Surface antigen was transformed into maize and this seed was used to evaluate expression levels through the breeding process. The transgene was transferred into two elite maize inbreds by backcrossing. Highest expressing ears were selected each generation until approximately $99 \%$ commercial parent was obtained with a single gene coding for the vaccine present. Selected individuals were crossed to create hybrid plants. This work was done to create high expressing high yielding lines that could be used as a plant-based oral vaccine for Hepatitis B. 
LIST OF TABLES ..................................................................

LIST OF FIGURES.....................................................................

CHAPTER 1: Literature Review..............................................

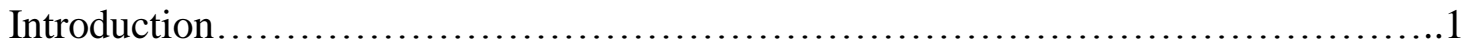

Plant Based Oral Vaccinations...........................................2

Hepatitis B Vaccines Currently Available...........................................4

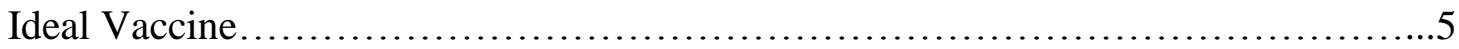

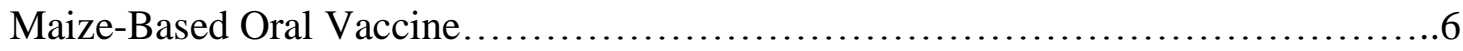

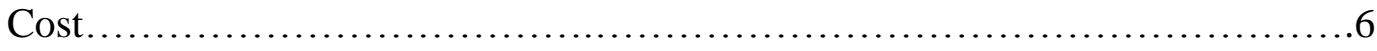

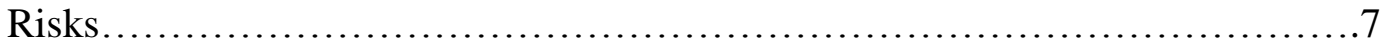

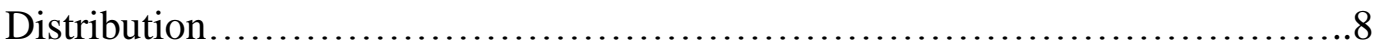

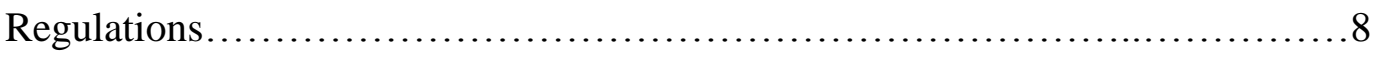

Plant Based Oral Vaccine Example.......................................9

Conclusion............................................................. 10

CHAPTER 2: Materials and Methods...............................................12

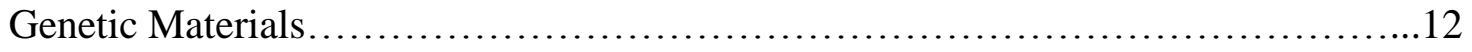

Greenhouse Production......................................................... 12

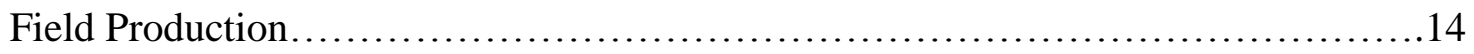

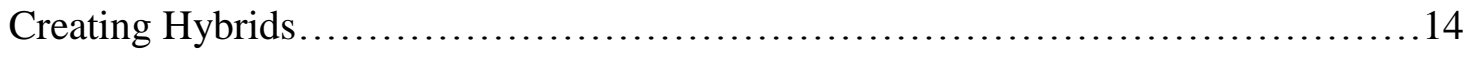

Pooled Seed Extracts.......................................................... 
HBsAg Seed Expression................................................ 15

Seed Weights..........................................................16

Statistics.................................................................

CHAPTER 3: Results......................................................... 17

Backcross Expression and Seed Weights - Female 16038 Parent..................17

Backcross Expression and Weight - Male Parent..............................19

Hybrids.................................................................20

Discussion.......................................................... 21

CC140097....................................................23

Hybrid program.......................................................24

CC140098 and CC140099..........................................24

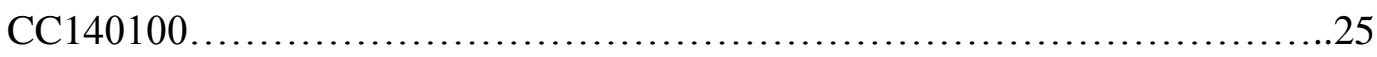

CHAPTER 4: Conclusions..................................................27

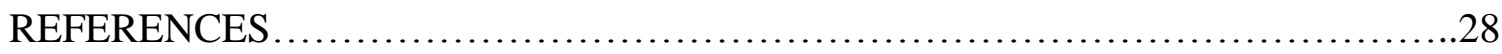




\section{LIST OF TABLES}

Table

Table 1: Expression in for the backcross program, and first self in the 16038 line.......... 18

Table 2: Seed weights for the backcross program, and first self in the 16038 line.......... 18

Table 3: $\mathrm{mg} / \mathrm{kg}$ for each cross of the backcross program in the MBS5411 line.............. 19

Table 4: Seed weights for each cross of the backcross program in the MBS5411 line.... 20

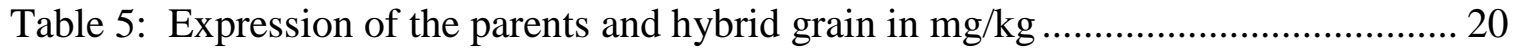

Table 6: Seed weights of parents and hybrid grain expressed in grams per 25 seed ....... 21 
Figure

\section{LIST OF FIGURES}

Page

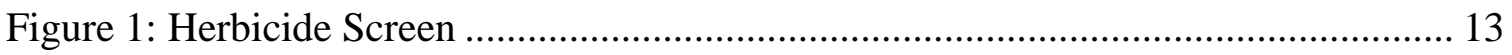

Figure 2: Expression in the 16038 line expressed in $\mathrm{mg} / \mathrm{kg}$ throughout the backcross... 22

Figure 3: Weight of 25 seed throughout the breeding program for the 16038 line........ 22

Figure 4: Expression in MBS5411 expressed throughout the backcross program .......... 23

Figure 5: Weight of 25 seed throughout the breeding program for MBS5411 ............... 24

Figure 6: Expression levels for the hybrid and the resulting hybrid grain ..................... 25

Figure 7: Seed weights that created the hybrid and the resulting hybrid grain................ 26 


\section{CHAPTER 1 \\ Literature Review}

\section{Introduction}

The hepatitis B virus (HBV) is a common virus, with two billion people infected worldwide. It causes approximately 600,000 deaths each year, despite the availability of an effective vaccine since 1982. Chronic infection with HBV can be life-threatening causing liver failure due to cirrhosis of the liver, and liver cancer (WHO, 2012). A heat stable oral vaccination would be easier to administer than a traditional vaccine in those areas where $\mathrm{HBV}$ is most prevalent. In developing parts of the world, vaccinations are not easily accessible; they require proper storage, safe vaccination conditions, and properly trained medical personnel. A method of vaccination that eliminates most of these problems could reduce the number of people who contract hepatitis B and suffer from the painful symptoms of a chronic or acute infection (WHO, 2012).

Diagnosis is accomplished by blood tests, which may determine a chronic, or acute infection. Blood tests identify the hepatitis B surface antigen (HBsAg), indicating patients with an active infection. Acute infections have no specific treatment but maintaining balanced nutrition, and hydration may help ease symptoms. The core antigen $(\mathrm{HBcAg})$ is another indicator of infection; a positive core antigen test and $\mathrm{HBsAg}$ test usually suggests a chronic infection. Chronic infections can be treated with medications such as interferon and antivirals, but these are not practical in developing countries because of cost and lack of availability of the medication. When the virus causes liver cancer, surgery and chemotherapy can extend life for several years, but those in developing countries typically die soon after diagnosis. With infection levels around 
$30 \%$ worldwide a more practical solution must be found for vaccine administration (WHO, 2012).

\section{Plant Based Oral Vaccinations}

Plant based oral vaccine candidates are created by introducing DNA into the plant genome. The DNA contains sequences designed to express a protein that may function as a subunit vaccine. Plant-derived oral vaccines may be safer than traditional recombinant vaccines because they eliminate the risk of exposure to animal-derived contaminants. In the development of a vaccine, the subunit protein is considered a candidate vaccine and if approved will become a vaccine. Most vaccines require cold storage and injection equipment whereas some plant-based oral vaccines have shown heat stability eliminating the need for a cold chain (Lamphear, 2002).

Maize as a platform for oral vaccination can supply an efficacious powerful, successful vaccine, which is more stable in percent total soluble protein (\%TSP) and does not require syringes or trained personnel to administer (Lamphear, 2002). The maize seed anatomy allows an ideal environment for a vaccination because it is a naturally bioencapsulated environment with a high concentration of protease inhibitors, low moisture and high concentration of carbohydrates. These factors lead to stability during storage and allow some antigen to survive the gastrointestinal tract that results in an immunologic response. Encapsulation may also help with thermostability of the antigen, which in turn keeps transportation and storage costs low (Hayden, 2012a). Many different diseases could be greatly reduced with plant based oral vaccinations. But expression levels need to be high for effective oral vaccination (Kirk, 2005). 
Plant based oral vaccinations have been used to protect against systemic intoxication by Shiga toxin Type 2. The Shiga toxin, which is in the bacteria group of $E$. coli, causing kidney failure in children and hemolytic uremic syndrome in adults. The vaccine was produced by destabilizing Shiga toxin A2 (StxA2) using mutagens and a plant optimized StxA2 was produced using the polymerase chain reaction and transforming this construct into tobacco using Agrobacterium-mediated transformation. Each plant was screened for the expression of this toxoid. Once toxoid expression was at high enough levels, mice were fed the oral vaccine and screened for the presence of a response by analyzing the immunoglobulin A ( $\operatorname{IgA})$, an antibody in the mucosal linings, and immunoglobulin $\mathrm{G}(\operatorname{IgG})$, an antibody in the blood. The immunoglobulin levels in the mice that received the vaccine determine if the vaccine triggered a response. This oral delivery method of a plant produced candidate vaccine was shown to be effective against the Shiga toxin (Wen, 2006).

A plant-based oral vaccine for hepatitis B has been produced in maize, with the maize producing the hepatitis B surface antigen in the embryo. Hayden et al created a construct, designated HBE, with a globulin 1 promoter sequence, which expresses preferentially in the embryo with expression levels of over $0.08 \%$ TSP from single seed analysis. Another backcross was performed, this time to an elite line to introduce commercial traits. Each plant was then self-pollinated for two generations to create homozygous lines that were used to create hybrids. The \% TSP of individual hybrid seed was as high as $0.46 \%$ and once bulked, expression was $0.20 \%$ TSP. These results were based on whole seed estimates but since the protein production was enriched in the embryo, removing the embryo from the whole seed created a more concentrated product. 
After testing just the embryo, dry weight concentration increased 6-fold, ultimately creating a large increase when comparing whole seed to embryo. When administered to mice, serum IgG showed a large increase with each boost as is expected to see with the injected candidate vaccine. This research shows the usefulness of recombinant technology as well as breeding and processing for the production of a heat stable, effective vaccine against hepatitis B (Hayden, 2012b).

A heat stable plant-based oral vaccine could greatly reduce the number of people infected with HBV. Molecular constructs were made to express HBsAg within the seed. Research by Hayden et al (2012a) showed that through plant breeding, the expression level can be increased to levels that produce an immune response in mice. A maize variety that has high expression of the HBsAg antigen can be used to safely deliver the hepatitis B vaccine to many parts of the world that have difficulty giving standard injections.

\section{Hepatitis B Vaccines Currently Available}

Currently there are several registered vaccines for hepatitis B in the United States, two for children and the other a combination vaccine for Hepatitis A and B safe only for adults. Engerix-B is registered from birth through 19 years old with a series of 3 injections intramuscularly at 0,1 , and 6 months with a dose of 10 micrograms $(\mu \mathrm{cg})$ (FDA, 1989). Adults over 19 years old should be given three injections of a higher dose of $20 \mathrm{mcg}$ at 0,1 , and 6 months. For those at higher risk of exposure to the virus an additional dose at 12 months can be added. Allergic reactions have been reported and they vary from anaphylaxis, fainting, and apnea with the most common problem being 
soreness at the injection site and fatigue. The preferred injection site is the front-outside part of the thigh for infants and for older children the deltoid is used. This injection is not to be used in the gluteral regions since an adverse response may occur (FDA, 1989).

Recombivax $\mathrm{HB}$ is another vaccine registered for both adults and infants and is a non-infectious subunit viral vaccine in the protein coat. To make this vaccine, HBsAg is cloned into yeast and the vaccine is produced from cultures of the recombinant yeast strain. The antigen is harvested and then undergoes a fermentation process which involves Saccharomyces cerevisiae which has the genes for the adw subtype of HBsAg and grows in yeast, soy peptone, dextrose, amino acids, and mineral salts. After more purification the final dose contains less than $1 \%$ yeast protein (Merck, 1983). A benefit to using this method is that the final product does not contain human blood or blood products and each batch is tested for sterility. There are three dosage levels: pediatric/ adolescent, adult, and dialysis. The pediatric/ adolescent dose is $10 \mathrm{mcg}$, and the adult dose is $20 \mathrm{mcg}$. This vaccine is to be injected intramuscularly with a 3 dose schedule of month 0,1 , and 6 . Adolescents age 11-15 can receive a 2 dose regime with a higher dose at month 0, and 4-6 months later (Merck, 1983).

\section{Ideal Vaccine}

An ideal vaccine must have each of the following traits: low cost, ease of administration, high safety profile, high potency, ease of storage and shipping, stability at room temperature, and low allergenicity. Maize as an oral vaccination is very promising, due to its high digestibility in humans, and its low potential as an allergen (Hayden, 2012b). It is highly unlikely that introducing one gene would cause maize to be more 
allergenic than it is currently (Ramessar, 2008). An allergic reaction to maize is rare and only occurs in a small percentage of humans but the reaction can be severe (Food, 2013). Traditional yeast-derived vaccines may also cause a severe allergic reactions in approximately one in 600,000 doses (Offit, 2013).

Maize can be grown in most parts of the world which allows for regions to grow their own crop to match local demand for a plant-based pharmaceutical. Maize grows quickly, has high yields, can be shipped easily without the need for cold storage, and maize has 'generally regarded as safe' (GRAS) status by the Food and Drug Administration (FDA) (Ramessar, 2008). Maize may also have multiple antigens inserted into the genome allowing for more than one pathogen to be targeted. Alternatively, many different maize lines can be blended to combine different antigens in one maize bulk to create multi-use oral vaccines (Lamphear, 2002).

\section{Maize-Based Oral Vaccine}

\section{Cost}

Vaccines could be grown in commercially available maize lines, allowing for large scale production, reducing total cost. Antigens expressed in the seed may remain stable inside the grain, removing need for refrigeration. The potential exists that a combination of transgenic lines could be created to produce a combination vaccine (Streatfield, 2001). With a combination vaccine fewer items would be shipped, refrigerated, and administered, further lessening cost. Another way to reduce the cost is to lessen or delete the need for the cold chain. Maintaining cold storage costs the world 200-300 million dollars per year (Giudice, 2006). As an example, avidin, a protein in 
eggs that binds biotin and is used in many diagnostic tests, was genetically inserted into maize. The protein produced in plants was equivalent to that found in chicken eggs but at a much lower cost than production in eggs. The grain could be stored for years without losing activity and because the animal was removed there is no chance of contaminating workers with salmonella (Howard, 2005).

Risks

One common concern is the potential contamination of the food supply with products that are not intended for the food supply. Both the Food and Drug Administration (FDA) and the United States Department of Agriculture (USDA) have compiled a set of regulations designed to maintain food safety with an increase in the production of plant-based pharmaceutical crops. Other possible risks include the allergenic potential of maize, and how the introduction of the transgene may produce different post translational products that may produce a new allergen. The Cartagena Protocol on Biosafety was created and adopted by many countries to standardize risk management for many products including plant based oral vaccines (Nap, 2003). Vertical gene transfer is avoided by having large buffer areas between pharmaceutical crops and food crops of the same species. Regulations state that a pharmaceutical crop must be at least one mile away from another crop of the same species (Becker, 2005). This buffer zone is effective in maize because maize has short-lived pollen, a short dispersal range, and no wild relatives in North America (Ramessar, 2008). 


\section{Distribution}

Plants store proteins naturally, by using tissues of the plant that are able to store high amounts of protein without degradation would reduce the need for refrigeration, thus reducing transportation and storage costs (Streatfield, 2001). The drying of seed results in the long term storage ability at ambient temperatures (Rybicki, 2009). Hayden et al stored maize seed with $\mathrm{HBsAg}$ at different temperatures for a month at $-20^{\circ} \mathrm{C}$ and $55^{\circ} \mathrm{C}$ and found no decrease in expression with oil-extracted samples and the conclusion was made that $\mathrm{HBs} \mathrm{Ag}$ does not decrease in oil-extracted samples at $55^{\circ} \mathrm{C}$ for an extended amount of time (2012 a). Also, there are fewer problems with biological safety since there are no viral or human pathogens in plants therefore only simple storage is needed with no need for cold storage (Shchelkunov, 2010).

\section{Regulations}

The USDA oversees the production and transport of transgenic material, with a focus on minimizing genetic transfer, done through containment. To prevent genetic transfer buffer zones are kept between transgenic and non-transgenic crops of the same variety, eliminating the possibility of cross pollination. If the physical buffer zone cannot be achieved, a temporal buffer can be created by planting weeks apart so the reproductive stage of one field would be different than the other. At harvest this is maintained by utilizing separate equipment for transgenic and non-transgenic crops as well as separate secure locations for storage, and transportation (Kirk, 2005).

The FDA regulates the pharmaceutical aspect of the crop in testing, creating the product, and the sale of the edible vaccine. Good Laboratory Practices (GLP) and Good 
Manufacturing Practices (GMP) have been created to merge both risk management practices and production methods to reduce the possibility of contamination and to minimize risks of the pharmaceutical material getting into a food source. Both the FDA and USDA will have to work in conjunction to cover the different sectors that each branch oversees (Kirk, 2005).

\section{Plant-Based Oral Vaccine Example}

An edible subunit vaccine against strains of enterotoxigenic $E$. coli was created in maize. The LT-B gene of E. coli and cholera toxin B have proven to confer protection against $E$. coli in mice. Transformations were done to immature embryos of Hi-II and the $\mathrm{T}_{0}$ plants were planted in a greenhouse (Streatfield, 2002). Hi-II is a line of maize that is known to have good embryos for transformation but low agronomic qualities (Armstrong, 1991). $T_{1}$ seed was planted in a field and a selection spray of $1 \%$ glufosinate ammonium was used to separate the transgenic plants from the non-transgenic plants. The $T_{1}$ plants were crossed with two elite inbred lines, one a Lancaster and the other a Stiff Stalk to produce $T_{2}$ lines. Hybrids were created when the two elite lines containing the transgene were crosses. To determine the amount of vaccine present in the seed, one seed was ground, and an ELISA assay conducted to determine the percent of total soluble protein (\%TSP) which translates into the concentration of antigen present in the seed. Through plant breeding the lines improved in both agronomic traits and the amount of vaccine present. The $\mathrm{T}_{1}$ lines expressed 0.013 to $1.8 \% \mathrm{TSP}$ while the $\mathrm{T}_{3}$ line expressed $9.2 \%$ TSP, a five-fold increase (Streatfield, 2002). 


\section{Conclusion}

Hepatitis B is easily transmitted through blood, bodily fluids, perinatal infection, sexual contact, and unsafe injections. HBV is 50 to 100 times more infectious than HIV, and can survive outside the body for seven days. Once the virus infects a host, there is typically an incubation period of 90 days. To prevent the spread of hepatitis B, children are vaccinated within the first day of life, with a typical vaccination administered in three doses. Following vaccination, children show a 95\% antibody response while adults show a 90\% antibody response. When those individuals who are non-responders are given an extra boost 30-50\% of them become responders (Kwon, 2011). Once vaccinated, the protection provided lasts at least 20 years and possibly lifelong. Currently 179 of 196 countries have hepatitis B as part of their vaccination schedules, which in many countries has reduced the rate of chronic infections in children to $1 \%$ compared to an 8-15\% infection rate before the vaccination (WHO, 2012). Many developing countries are not able to administer the vaccination because it is expensive, and requires cold storage. They also lack the ability to administer critical booster vaccinations, as well as provide trained personnel to administer the injection following sterile procedures.

An acute infection presents itself with jaundice, dark urine, tiredness, nausea, vomiting and abdominal pain while those who have an advanced chronic infection develop cirrhosis of the liver or liver cancer. Children are the most susceptible to the hepatitis B virus when infected during their first year, with 90\% developing chronic infections. Children between the ages one to four develop chronic infection at a rate of 
$30-50 \%$. Of the children infected, $25 \%$ develop liver cirrhosis or cancer and die in adulthood (WHO, 2012).

Vaccines can be created using recombinant gene technologies in which the vaccine can be made without a human or animal host and only includes the antigen needed. Recombinant vaccines however, still have the potential for contamination from mammalian cell culture. The development of a new type of vaccine that does not use animals or needs to be injected would be a benefit in terms of cost and administration hurdles. A plant-based oral vaccine is a reasonable alternative. Plants produce proteins at a level high enough to vaccinate and plants are not susceptible to animal viruses that could infect the recipient. Therefore, creating a plant based oral vaccine that is similar to current vaccines and eliminates the need for needles is possible. 
CHAPTER 2

\section{Materials and Methods}

\section{Genetic Materials}

Two Stine Inbred lines 16038, a female stiff stalk type, and MBS5411, a Lancaster male type, were chosen because of high combining ability of the parents producing high yielding, uniform lines when crossed. 16038 is a high yield, and yellow dent variety. MBS5411 is a high kernel weight, large ear diameter, yellow dent variety. Material expressing Hepatitis B surface antigen was created and obtained from the Applied Biotechnology Institute. The lines used were crossed once to Hi-II and prior to starting the backcross program. After each backcross, highest expressing ears were selected and carried forward into the next generation. The transgene was backcrossed 5 or 6 times into both lines until approximately $99 \%$ commercial parent was obtained with a single gene coding for the vaccine present. The 16038 line at backcross 4 (BC4) had sibling lines that were chosen to continue the program for further backcrossing and an accelerated hybrid, these lines are designated 16038-1 and 16038-2. To determine whether the gene coding for the vaccine is present the gene of interest is linked to herbicide resistance, with herbicide screening done each generation.

\section{Greenhouse Production}

Backcrossing of the transgene into elite inbred lines was completed in greenhouses on the campus of California Polytechnic State University in San Luis Obispo, California. Plants were grown in containers using a Foxfarm ocean forest 1.5 cubic foot (cf) potting soil and $64 \mathrm{oz}$. of Aztec perlite. All Pots were autoclaved for 25 minutes or bleached using a $10 \%$ solution to remove insects and pathogens. Amount of 
seed planted per pot was determined by percent germination from the previous generation and number of ears needed at the end of the study. Number of seeds germinated was recorded. At the 3-to-4 leaf stage, plants were screened for the presence of the gene of interest utilizing the glufosinate-ammonium resistance screen. Resistance screen in the greenhouse was done using a leaf paint technique so as to not kill the recurrent parent that are not resistant to the herbicide. The leaf paint technique is done by marking an $\mathrm{X}$ on a leaf and herbicide applied with a cotton swab. Five days after painting, leaf results of the herbicide screen were taken by counting susceptible and resistant plants. Susceptibility was determined by a browning of the leaf tip and necrosis across the painted section of the leaf (Figure 1). No reaction to the herbicide was counted as resistance. Susceptible plants were removed from pots and resistant plants were allowed to continue growth.
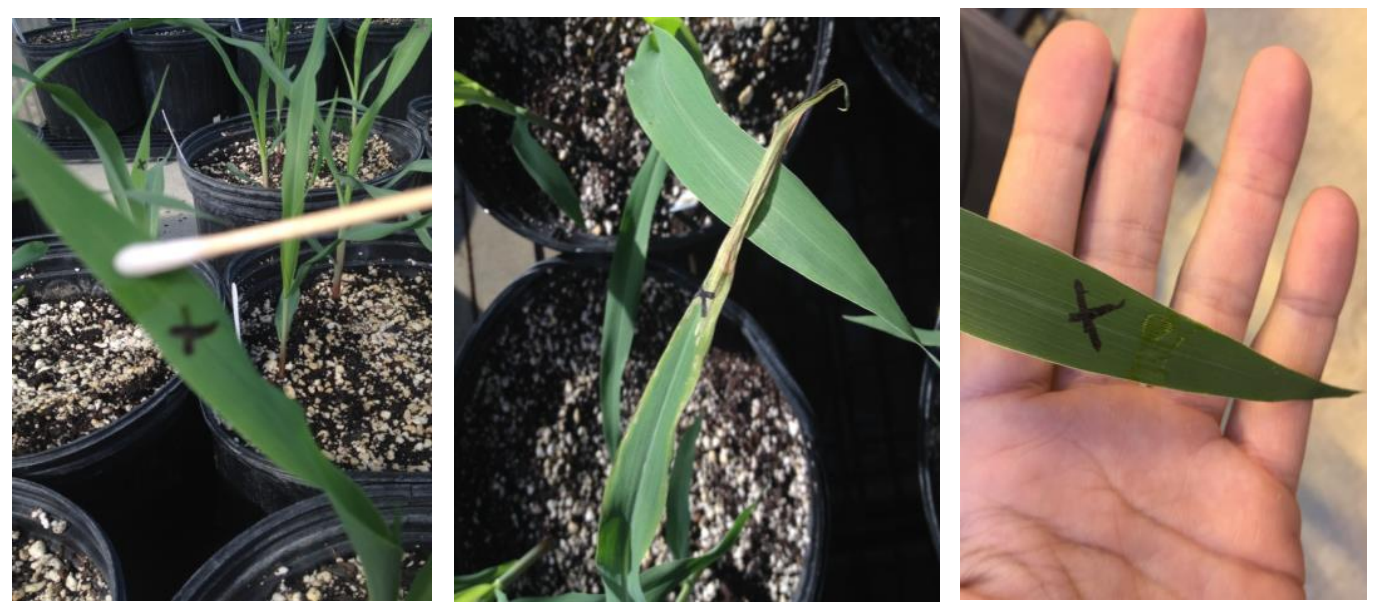

Figure 1: Placement of the herbicide (left), a plant showing a susceptible response (middle), and a plant showing a resistant response (right)

Pollinations were done following the procedure described previously (Smith, 2004). Thirty two days after pollination the ear was harvested and dried in an oven at $37.0^{\circ} \mathrm{C}$ until the seed was approximately $15 \%$ moisture. After shelling the seed was sent to the lab for analysis. 


\section{Field Production}

The field production of transgenic maize was done on an approximately one acre plot. The maize was grown following conventional agriculture practices. Percent germination for each line was determined shortly after emergence. Herbicide screening was conducted on all transgenic lines with a backpack sprayer with $1.0 \%$ of active ingredient herbicide solution of glufosinate-ammonium at the three to four leaf stage. The herbicide screen was conducted with a backpack sprayer and shield to avoid spraying the recurrent parents, which are susceptible to the herbicide. Plants susceptible to the herbicide were killed all resistant lines were assumed to contain the transgene and allowed to continue growth. All plants containing the gene of interest were either backcrossed or selfed depending on their stage in the program. Three backcrosses were completed in the field 16038-1-BC1, 16038-1-BC6, MBS5411-BC1 and all hybrids were grown in the field. All pollinations were done according to the procedure described previously (Smith, 2004). Ears were harvested and dried to 12-14\% moisture, and shelled. Line weight was recorded and seed was sent to the lab for analysis of the HBsAg concentration.

\section{Creating Hybrids}

The 16038 and MBS5411 lines containing the recombinant protein were crossed to create hybrid seed and then the hybrid seed was planted in the field and allowed to open pollinate creating hybrid grain. CC140097 was a cross between 16038-2- BC5 and MBS5411-BC4. CC140098 and CC140099 lines were both created by the cross 16038 - 
1-BC6 and MBS5411-BC5. Lines were split after analysis into a high expressing and low expressing bulk. Line CC140100 was the only line with one self and was a cross between 16038-2-BC5S1 and MBS5411-BC5. Ears were allowed to dry-down on the plant, hand-harvested from the field, and analyzed.

\section{Pooled Seed Extracts}

Seed from the backcross program was pooled from a single ear and 50 seeds were randomly selected. For hybrid seed and hybrid grain all ears were bulked from a single line and the 50 seed pool was taken from the bulk. Bulked seed was ground in a Speck Mill, Coffee Grinder for approximately 30 seconds. $100 \mathrm{mg}$ of ground material was weighed in an extraction vial, a 3/8" ball bearing was placed on top of the maize, and $1 \mathrm{ml}$ of extraction buffer of ( $1 \mathrm{~mL}$ phosphate buffer (PBS) and $1 \%$ Triton $\mathrm{X})$ was added to each vial and capped. The vial was then shaken for 20 seconds at a rate of 600 strokes per minute in a GENO/GRINDER 2000. Samples were then centrifuged using a Avanti J25 (Beckman Allegra, Brea Ca.) with a swinging bucket rotor JS-5.9 at 2,000 RCF (3280 RPM) and $4^{\circ} \mathrm{C}$ for $13-17$ minutes. $230-240 \mu 1$ of the supernatant was removed and placed in a 96-well plate on ice.

\section{HBsAg Seed Expression}

After each generation of seed was produced, the seed was analyzed to determine milligram of HBsAg per kilogram of maize material $(\mathrm{mg} / \mathrm{kg})$. The testing procedure from the start of the project until 2013 has been described previously (Hayden, 2012a). In 2013 the procedure changed and the modifications are described by Hayden et al 
(2014). To account for any variation in the different procedures all the seed was rerun after the hybrid grain was harvested.

An ELISA assay was done using 100 milligrams (mg) of ground maize and extractions were done in 1 milliliter of PBS and 1\% Triton-X 100. The ELISA was done with dilutions 1:250 as described by Hayden (2014). The ELISA assay were measured with an induced chromophore response at 405nm (Hayden, 2012a).

\section{Seed Weights}

25 seed weights were taken by counting out 25 seed from each bulked seed. The seed was weighed in grams to the hundredths place. Three different 25 seed samples were taken from each individual bulk and averaged.

\section{Statistics}

Two-sample t-tests were conducted between each generation; significant differences between expression at different levels of backcrossing were identified. Twosample t-tests, were performed on Minitab to determine significant differences among HBsAg expression and 25 seed weights. The alpha level for each test was 0.10 . 


\section{CHAPTER 3}

\section{Results}

\section{Backcross Expression and Seed Weights - Female 16038 Parent}

The transformed line was backcrossed (BC) to the female parent 16038 for five or more generations depending on the line. Herbicide screening was accomplished each generation, with hepatitis B surface antigen expression levels evaluated each generation. Expression levels from BC1 through BC4 were 89.0, 70.0, 37.0, and $130 \mathrm{mg} / \mathrm{kg}$ respectively (Table 1). The BC4 line was backcrossed once more to 16038 to produce several sibling ears. Two of the highest expressing sibling ears of the $\mathrm{BC} 4$ were chosen to continue the program but take different paths, these lines are designated 16038-1 and 16038-2. 16038-1-BC5 had an expression of $129 \mathrm{mg} / \mathrm{kg}$, which was backcrossed again and 16038-1-BC6 had an expression of $82 \mathrm{mg} / \mathrm{kg}$. 16038-2-BC5 had an expression of 99 $\mathrm{mg} / \mathrm{kg}$ and the $16038-2-\mathrm{BC} 5 \mathrm{~S} 1 \mathrm{had}$ an expression of $222 \mathrm{mg} / \mathrm{kg}$. The only lines that showed a significant difference were between BC2 and BC3 $(p=0.067), B C 3$ and BC4 $(\mathrm{p}=0.078)$, BC5 and BC5S1 ( $\mathrm{p}=0.047), 16038-1-\mathrm{BC} 5$ and 16038-1-BC6 $(\mathrm{p}=0.004)$.

Seed weights from the backcrossed lines for BC1 through BC4 were 6.06, 2.80, 3.68, and 3.71 grams respectively. 16038-1 BC5 had a seed weight of 3.53 grams and 16038-1-BC6 had a seed weight of 5.23 grams. 16038-2-BC5 had a seed weight of 3.36 grams and the 16038-2-BC5S1 seed weight of 5.19 grams. (Table 2). Significant differences were identified between 16038-1-BC1 and 16038-1-BC2 ( $\mathrm{p}=0.001), 16038-1$ BC2 and 16038-1-BC3 (p =0.003), and 16038-1-BC5 and 16038-1-BC6 ( $\mathrm{p}=0.004)$. A difference in weight between 16038-1-BC4 and 16038-2-BC5 (p=0.092), and 16038-2BC5 and 16038-2-BC5S1 ( $\mathrm{p}=0.019)$. 
$16038-1 \quad 16038-2$

\begin{tabular}{c|cr} 
BC1 & 89.0 \\
BC2 & 70.0 & \\
BC3 & 37.0 & \\
BC4 & 130 & \\
BC5 & 129 & 99 \\
BC5S1 & - & 222 \\
BC6 & 82 & - \\
\hline
\end{tabular}

Table 1: Expression in $\mathrm{mg} / \mathrm{kg}$ for each cross of the backcross program, and first self in the 16038 line.

16038-1 16038-2

\begin{tabular}{l|ll}
\hline BC1 & 6.06 \\
BC2 & 2.8 \\
BC3 & 3.68 \\
BC4 & 3.71 & \\
BC5 & 3.53 & 3.36 \\
BC6 & 5.23 & - \\
BC5S1 & - & 5.19 \\
\hline
\end{tabular}

Table 2: Seed weights in grams for each cross of the backcross program, and first self in the 16038 line. 


\section{Backcross Expression and Weight - Male Parent}

The male parent MBS5411 was backcrossed for five generations. Herbicide screening was accomplished each generation, with expression levels evaluated each generation. Expression levels from BC1 through BC4 were 33.3, 191, 240, 188, and $173.5 \mathrm{mg} / \mathrm{kg}$ respectively (Table 3 ). The only significant difference in surface antigen expression was between $\mathrm{BC} 1$ and $\mathrm{BC} 2(\mathrm{p}=0.043)$ at a $\mathrm{p}$-value of 0.10 . All other backcrosses had no significant differences observed.

MBS5411

\begin{tabular}{l|l}
\hline BC1 & 33.3 \\
BC2 & 191 \\
BC3 & 240 \\
BC4 & 188 \\
BC5 & 173 \\
\hline
\end{tabular}

Table 3: Expression in $\mathrm{mg} / \mathrm{kg}$ for each cross of the backcross program in the MBS5411 line.

Seed weights from the backcrossed lines for MBS5411-BC1 through MBS5411BC5 were 5.58, 4.09, 5.41, 2.85, and 4.77 grams respectively (Table 4). Significant differences were found between all backcross generations, MBS5411-BC1 and MBS5411-BC2 (p=0.017), MBS5411-BC2 and MBS5411-BC3 (P=0.017), MBS5411BC3 and MBS5411-BC4 (p=0.002), and MBS5411-BC4 and MBS5411-BC5 ( $\mathrm{p}=0.004)$. 


\section{MBS5411}

\begin{tabular}{|l|r|}
\hline BC1 & 5.58 \\
\hline BC2 & 4.09 \\
\hline BC3 & 5.41 \\
\hline BC4 & 2.85 \\
\hline BC5 & 4.77 \\
\hline
\end{tabular}

Table 4: Seed weights in grams for each cross of the backcross program in the MBS5411 line.

\section{Hybrids}

Four hybrid lines were developed to evaluate the hybrid performance at different levels of backcross generations. The hybrid grain CC140097 had a total expression of $99.2 \mathrm{mg} / \mathrm{kg}$ (Table 5) and a seed weight of 7.53 grams (Table 6). Hybrid CC140098, the high expressing bulk, expressed $129 \mathrm{mg} / \mathrm{kg}$ with a seed weight of 8.53 grams and CC140099, the low expressing bulk, expressed $80.8 \mathrm{mg} / \mathrm{kg}$ with a seed weight of 7.75 grams. Hybrid CC140100 expressed $82 \mathrm{mg} / \mathrm{kg}$ and seed weight was 8.09 grams.

\begin{tabular}{|c|c|c|c|c|}
\hline & CC140097 & CC140098 & CC140099 & CC140100 \\
\hline $\begin{array}{c}\text { MBS5411 } \\
\text { PARENT }\end{array}$ & 188 & 173 & 173 & 173 \\
\hline $\begin{array}{c}\text { 16038 } \\
\text { PARENT }\end{array}$ & 99 & 82 & 82 & 222 \\
\hline HYBRID & 99.2 & 129 & 80.8 & 81.1 \\
\hline
\end{tabular}

Table 5: Expression of the parents and hybrid grain in $\mathrm{mg} / \mathrm{kg}$ 


\begin{tabular}{|c|c|c|c|c|}
\hline & CC140097 & CC140098 & CC140099 & CC140100 \\
\hline $\begin{array}{c}\text { MBS5411 } \\
\text { PARENT }\end{array}$ & 2.85 & 4.77 & 4.77 & 4.77 \\
\hline $\begin{array}{c}\text { 16038 } \\
\text { PARENT }\end{array}$ & 3.35 & 5.23 & 5.23 & 3.84 \\
\hline HYBRID & 7.53 & 8.53 & 7.75 & 8.09 \\
\hline
\end{tabular}

Table 6: Seed weights of the parents and hybrid grain expressed in grams per 25 seed

\section{Discussion}

Expression was expected to fluctuate at the start of the backcrossing program but was expected to stabilize as backcrossing progressed. The hybrid grain was expected to increase in expression with increased seed size and increased positive alleles.

Unfortunately none of the hybrid parents in all hybrid crosses in this study were homozygous for the transgene. Backcross seed weight was expected to decrease as the backcross program progressed due to inbreeding while hybrid seed weight was expected to increase due to heterosis. Some of the fluctuations in expression levels may appear larger than they are due to the inaccuracy of the assay used. When tested the validated assay is $\pm 25 \%$.

Female- 16038

The female line increased expression in the $\mathrm{BC} 1(89.0 \mathrm{mg} / \mathrm{kg})$ and began to decrease in the $\mathrm{BC} 2$ and $\mathrm{BC} 3(70.0 \mathrm{mg} / \mathrm{kg}$ and $39.0 \mathrm{mg} / \mathrm{kg}$ respectively) before increasing in the BC4 (130.0 mg/kg) (Figure 2). At the BC4 the line was split into two 16038-1-BC5 and 16038-2-BC5. Seed weights followed the expected pattern (Figure 3). All lines were grown in difference environments with the BC3 seed the only seed grown in the winter greenhouse which relied on a large amount of artificial light leading to an 
alteration in growth, possibly contributing to the decreased expression. However, in this study we did not attempt to quantify the variability in expression due to environment.

The BC5S1 was expected to increase as the number of positive alleles in the line increased, thus increasing the total expression level. The BC5S1 was higher than BC5 going from $99.0 \mathrm{mg} / \mathrm{kg}$ to $222.0 \mathrm{mg} / \mathrm{kg}$. However, average seed weight remained the same between the two lines.

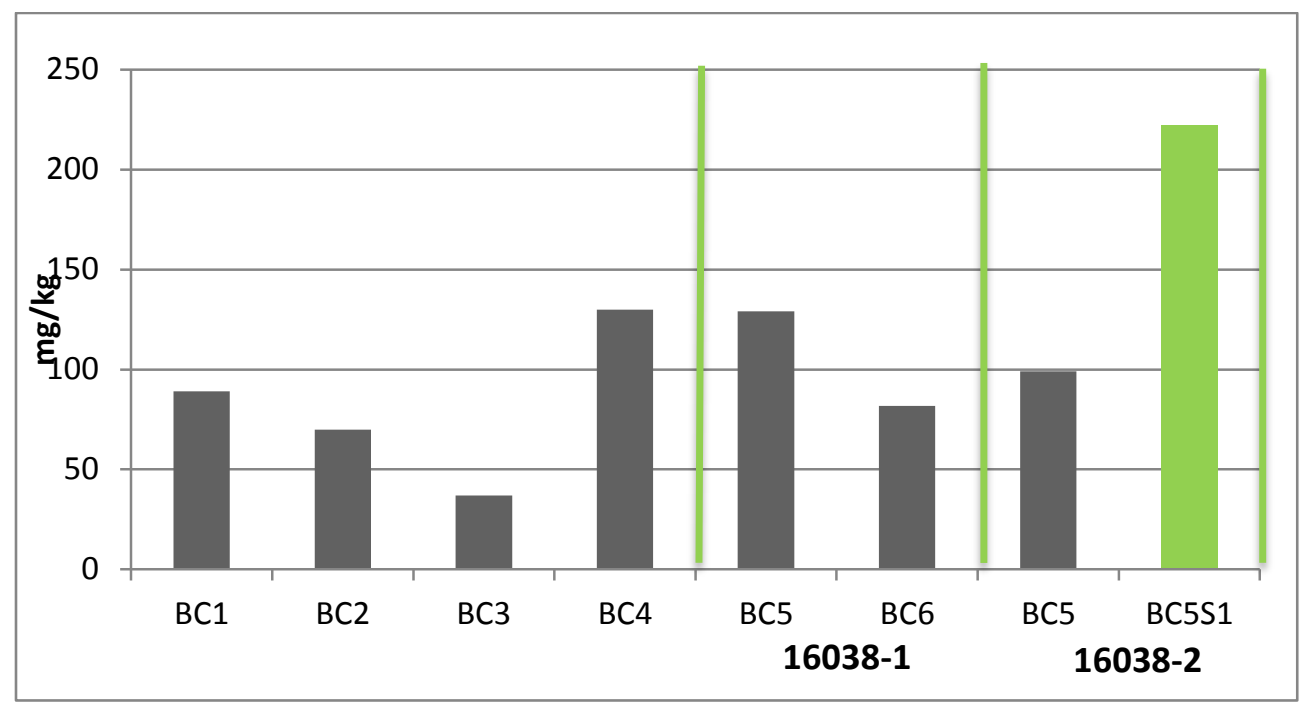

Figure 2: Expression in the 16038 line expressed in $\mathrm{mg} / \mathrm{kg}$ throughout the backcross.

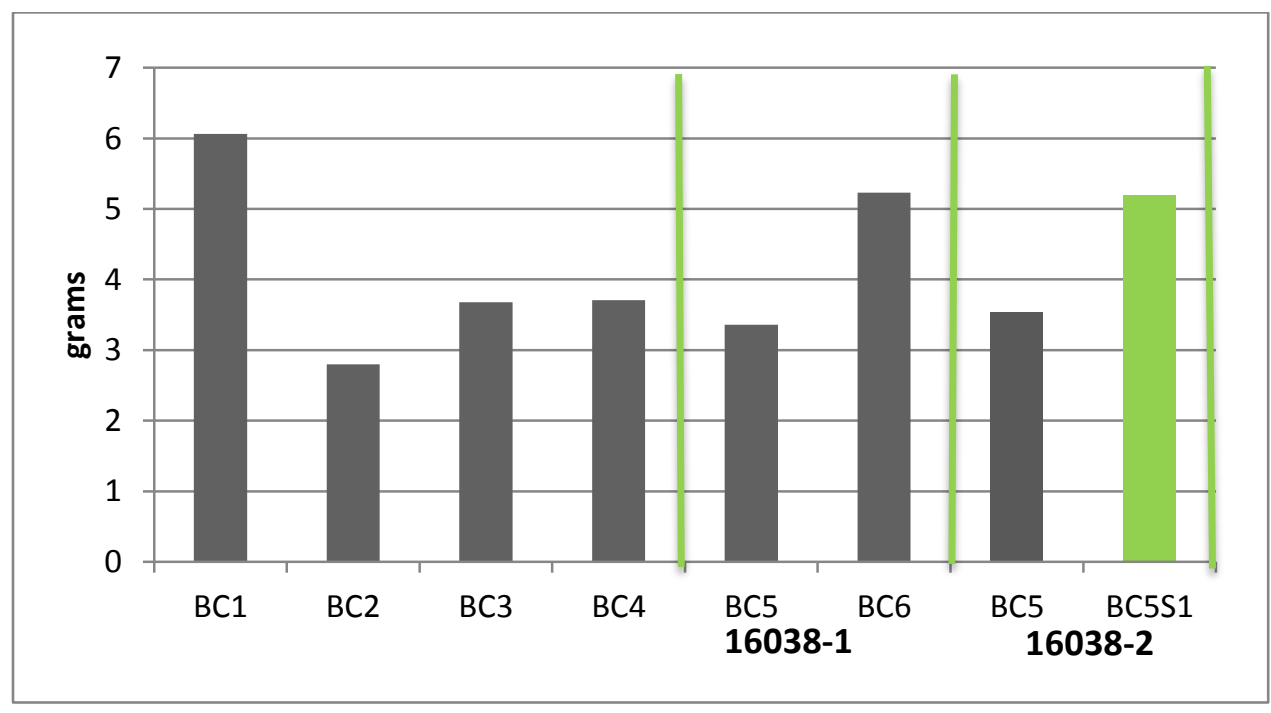

Figure 3: Weight in grams of 25 seed throughout the breeding program for the 16038 line. 


\section{Male-MBS5411}

The highest expressing male line was BC3 at $240 \mathrm{mg} / \mathrm{kg}$ (Figure 4). There was one significant difference in expression through the backcross program between $\mathrm{BC} 1$ $(33.3 \mathrm{mg} / \mathrm{kg})$ and BC2 $(191 \mathrm{mg} / \mathrm{kg})$ at a p-value of 0.048 . The seed weight of the line throughout the backcross program tapers off as the backcrossing continued. The low expression for $\mathrm{BC} 1$ can be contributed its early stage in the backcross program. $\mathrm{BC} 1$ seed was grown in the field while all other backcrosses were done in the greenhouse. $\mathrm{BC} 1$ material is $50 \% \mathrm{Hi}-\mathrm{II}$ which does not perform well in less than ideal growing conditions and so fluctuations in temperature and water may have contributed to its lower expression.

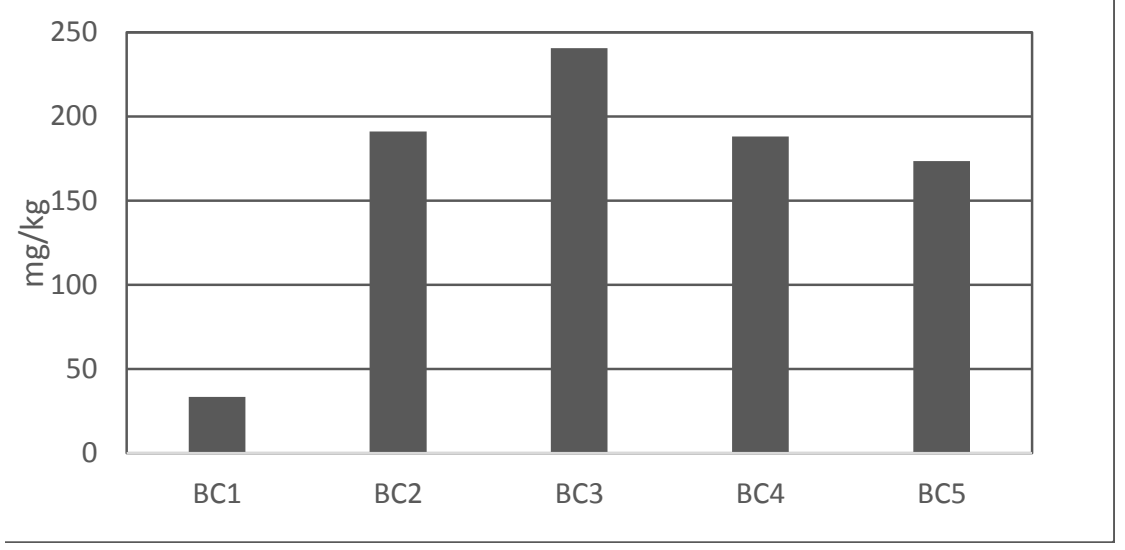

Figure 4: Expression in MBS5411 expressed in $\mathrm{mg} / \mathrm{kg}$ throughout the backcross program 


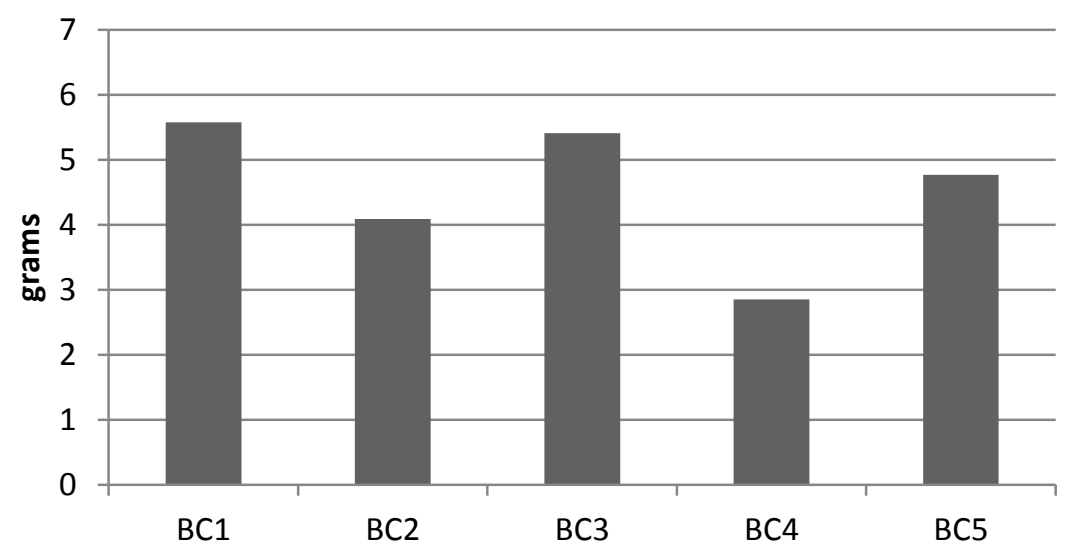

Figure 5: Weight in grams of 25 seed throughout the breeding program for MBS5411

\section{Hybrid program}

\section{CC140097}

Hybrid CC140097 was a cross of backcrossed individuals 16038-2-BC5 and MBS5411-BC4. Expression of the grain from this seed was an average of its two parents while seed weight increased (Figure 10 and 11). 16038-2-BC5 contains 98\% recurrent parent while MBS5411-BC4 contains 96\% recurrent parent. Seed weight increase was indicative of heterosis. The expression within the hybrid was lower than expected. This was likely due to a lack of homozygous parent lines leading to a segregating hybrid population. (Figure 11).

\section{CC140098 and CC140099}

Both CC140098 and CC140099 are a cross of 16038-1-BC6 and MBS5411-BC5. 16038-2-BC6 is 99\% recurrent parent and MBS5411-BC5 is 98\% recurrent parent. The hybrid seed was split into two bulks; a high expressing bulk (CC140098) and a low expressing bulk (CC140099) (Figure 10). The high expressing bulk expression was 
higher than the 16038 parent where the low expressing line was almost equivalent to the 16038 parent. The high and low expressing bulks performed as anticipated and suggests that selection of hybrid seed for expression prior to planting may increase total HBsAg concentration in the hybrid grain.

CC140100

Hybrid CC140100 is the only line with a self of the BC5. A self increases the percentage of homozygous individuals. With more homozygous individuals the dosage of the allele is increased. The hybrid grain was created from 16038-2-BC5S1 and MBS5411-BC5 line. The 16038-2-BC5S1 has 98\% recurrent parent and MBS5411-BS5 also has $98 \%$ recurrent parent. Unfortunately both parents were higher expressing than the hybrid grain (Figure 6). The hybrid grain weight showed that there was hybrid vigor in the grain but expression did not increase (Figure 7).

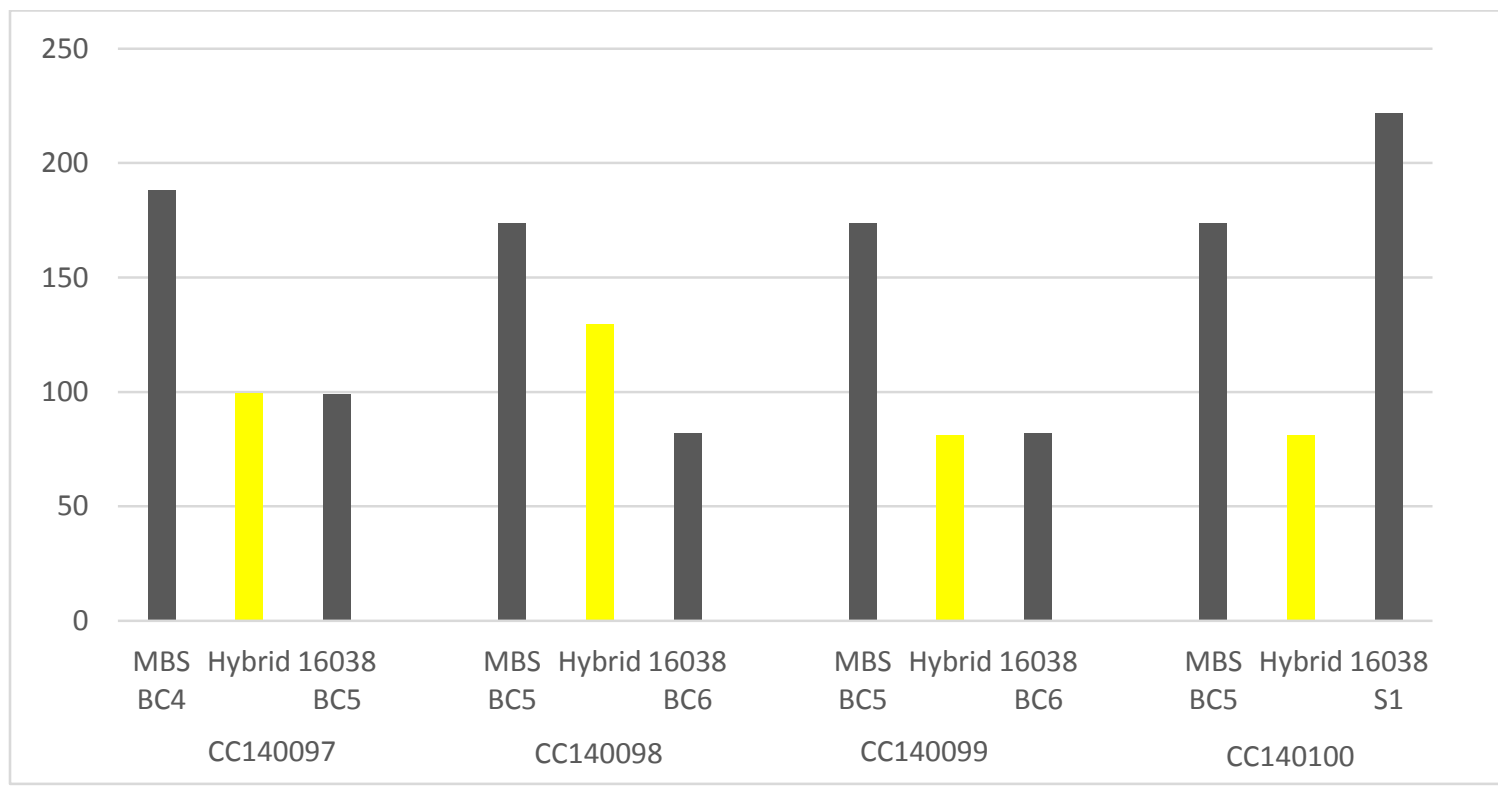

Figure 6: Expression levels for the lines that created the hybrid and the resulting hybrid grain in yellow. 


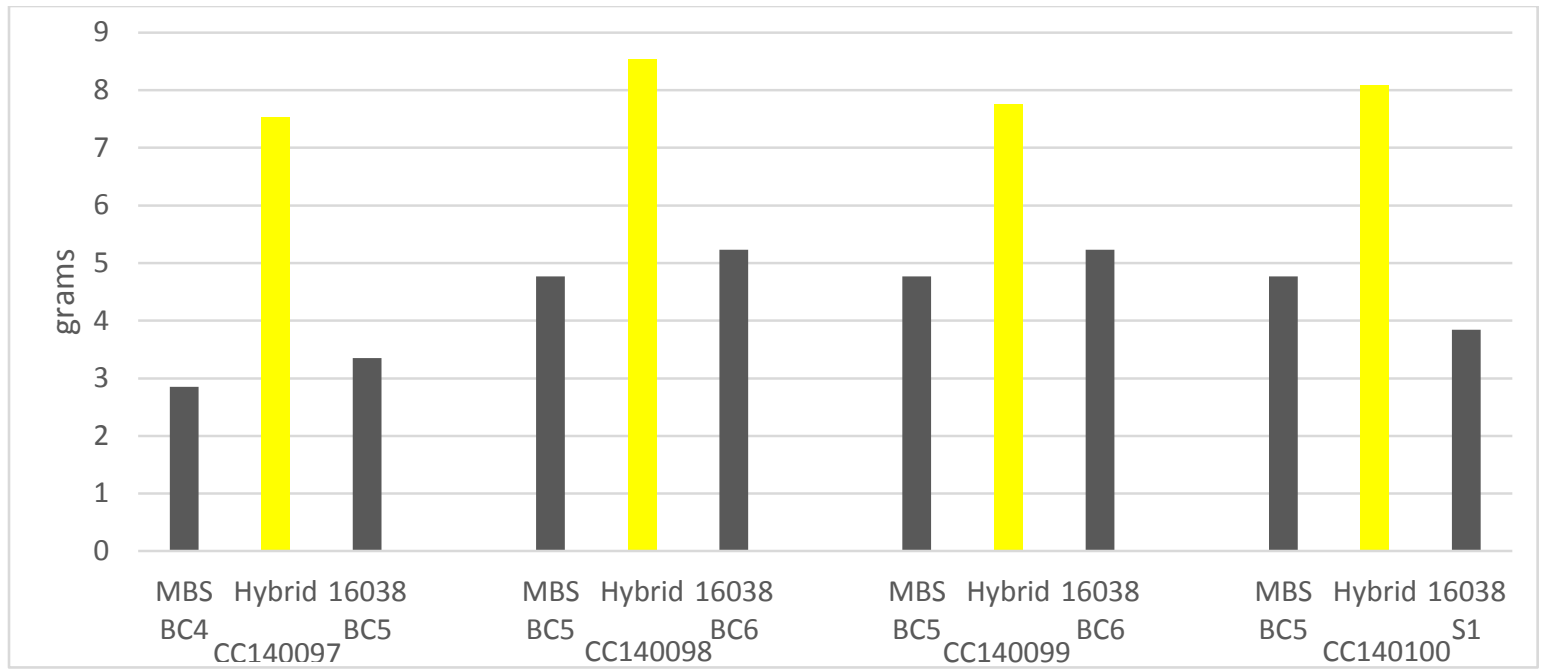

Figure 7: Seed weights for the lines that created the hybrid and the resulting hybrid grain in yellow. 


\section{CHAPTER 4}

\section{Conclusions}

The 16038 line showed expression fluctuation throughout the backcross program. The variable expression levels in the 16038 leads to the conclusion that environment plays a large role in expression of the transgene. Therefore more studies should be conducted to demonstrate if and how the environment influences expression. The transgene appears to express better in the MBS5411 line than the 16038. Therefore, a new elite female line that is compatible to MBS5411 may help decrease the fluctuation among the female line and increase expression in that line. With expression leveling off around backcross 5 and 6 further backcrossing may not be needed to increase expression and above $98 \%$ recurrent parent more backcrossing is not needed to improve agronomic traits. The hybrid grain expression is consistent with the parent lines not being homozygous while seed weights indicate heterosis in all lines. Further studies should be conducted with homozygous lines grown in the same environment to give a better indication of how genetics and the environment affect the expression of the hepatitis B surface antigen. A true hybrid is expected to show increased expression due to higher dosage of the transgene. 


\section{References}

(2013) Food Allergy Research and Education. About food allergies: Other allergens. Food Allergy Research and Education.

Armstrong C.L., Green C.E., Phillips R.L. (1991) Development and availability of germplasm with high Type II culture formation response.

Becker G.S., Vogt D. (2005) Regulation of Plant-Based Pharmaceuticals, The Library of Congress.

FDA. (1989) Highlights of Prescribing Information. Retrieved from:

http://www.fda.gov/downloads/BiologicsBloodVaccines/Vaccines/ApprovedPro ducts/UCM224503.pdf

Giudice E.L., Campbell J.D. (2006) Needle-free Vaccine Delivery, Advanced Drug Delivery Reviews. pp. 68-89.

Hayden C.A., Egelkrout E.M., Moscoso A.M., Enrique C., Keener T.K., Jimenez-Flores R., Wong J.C., Howard J.A. (2012a) Production of highly concentrated, heatstable hepatitis B surface antigen in maize. Plant Biotechnology Journal 10:979984. DOI: $10.1111 /$ j.1467-7652.2012.00727.x.

Hayden C.A., Streatfield S.J., Lamphear B.J., Fake G.M., Keener T.K., Walker J.H., Clements J.D., Turner D.D., Tizard I.R., Howard J.A. (2012b) Bioencapsulation of the hepatitis B surface antigen and its use as an effective oral immunogen. Vaccine 30:2937-2942. DOI: 10.1016/j.vaccine.2012.02.072.

Hayden CA, Smith EM, Turner DD, Keener TK, Wong JC, Walker JH, Tizard IR, Jimenez-Flores R, Howard JA (2014). Supercritical fluid extraction provides an enhancement to the immune response for orally-delivered hepatitis B surface antigen. Vaccine , doi: 10.1016/j.vaccine.2014.01.037

Howard J.A. (2005) Commercialization of Biopharmaceutical and Bioindustrial Proteins from Plants, Crop Science. pp. 468-472.

Kirk D.D., McIntosh K., Walmsley A.M., Peterson. R.K.D. (2005) Risk Analysis for Plant-made Vaccines, Transgenic Research. pp. 449-462.

Kwon S.Y., Lee C.H. (Epidemiology and prevention of hepatitis B virus infection) 2011, Journal of Viral Hepatitis. pp. 87-95. 
Lamphear B.J., Streatfield S.J., Jilka J.M., Brooks C.A., Barker D.K., Turner D.D., Delaney D.E., Garcia M., Wiggins B., Woodard S.L., Hood E.E., Tizard I.R., Lawhorn B., Howard J.A. (2002) Delivery of subunit vaccines in maize seed. Journal of Controlled Release 85:169-180. DOI:

http://dx.doi.org/10.1016/S0168-3659(02)00282-1.

Merck. (1983) Highlights of Prescribing Information. Retrieved from: http://www.merck.com/product/usa/pi_circulars/r/recombivax_hb/recombivax_pi .pdf

Nap J.-P., Metz P.L., Escaler M., Conner A.J. (2003) The release of genetically modified crops into the environment, The Plant Journal.

Offit, P. 2013. Can people with yeast allergies get vaccines? The Children's Hospital of Philadelphia.

Ramessar K., Sabalza M., Capell T., Christou P. (2008) Maize plants: An ideal production platform for effective and safe molecular pharming, Plant Science. pp. 409-419.

Rybicki E.P. (2009) Plant-produced Vaccines: Promise and Reality, Drug Discovery Today. pp. 16-24.

Shchelkunov S., Shchelkunova G. (2010) Plant-based Vaccines Against Human Hepatitis B Virus, Expert Review of Vaccines. pp. 947-955.

Smith W., Betran J., Runge E. (2004) Corn: Origin, History, Technology, and Production. John Wiley \& Sons. Pg. 335-356.

Streatfield S.J., Jilka J.M., Hood E.E., Turner D.D., Bailey M.R., Mayor J.M., Woodard S.L., Beifuss K.K., Horn M.E., Delaney D.E., Tizard I.R., Howard J.A. (2001) Plant-based Vaccines: Unique Advantages, Vaccine. pp. 2742-2748.

Streatfield S.J., Mayor J.M., Barker D.K., Brooks C., Lamphear B.J., Woodard S.L., Beifuss K.K., Vicuna D.V., Massey L.A., Horn M.E., Delaney D.E., Nikolov Z.L., Hood E.E., Jilka J.M., Howard J.A. (2002) Development of an Edible Subunit Vaccine in Corn Against Enterotoxigenic Strains of Escherichia Coli, In Vitro Cellular \& Amp. pp. 11-17.

W.H.O. (2012) Hepatitis B fact sheet, Online.

Wen S.X., Teel L.D., Judge N.A., O'Brien A.D. (2006) A plant-based oral vaccine to protect against systemic intoxication by Shiga toxin type 2. Proceedings of the National Academy of Sciences of the United States of America 103:7082-7087. DOI: $10.1073 /$ pnas.0510843103. 